\title{
Selective synthesis of Bisphenol-A over mesoporous MCM silica catalysts functionalized with sulfonic acid groups
}

\author{
Debasish Das, ${ }^{\mathrm{a}, 1}$ Jyh-Fu Lee, ${ }^{\mathrm{b}}$ and Soofin Cheng a,* \\ a Department of Chemistry, National Taiwan University, Taipei 106, Taiwan, ROC \\ ${ }^{\mathrm{b}}$ Research Division, National Synchrotron Radiation Research Center, Hsinchu 300, Taiwan, ROC
}

Received 13 October 2003; revised 19 January 2004; accepted 25 January 2004

\begin{abstract}
Mesoporous MCM-41 and -48 silicas anchored with sulfonic acid $\left(-\mathrm{SO}_{3} \mathrm{H}\right)$ groups via postsynthesis modification are very effective for the synthesis of Bisphenol-A by liquid-phase condensation of phenol with acetone. Higher amounts of thiol groups can be incorporated in MCM-48 silicas presumably due to the presence of larger number of surface silanol groups. However sulfur $K$-edge XANES spectroscopy reveals that effective oxidation of the precursor thiol $(-\mathrm{SH})$ groups to the sulfonic acid $\left(-\mathrm{SO}_{3} \mathrm{H}\right)$ groups was necessary for obtaining samples of good catalytic activity. It was noted that when sulfur loadings exceed $1.5 \mathrm{meq} / \mathrm{g}$ solid a part of the sulfur atoms remains in the reduced form even after prolonged oxidation. MCM-41 silica anchored with sulfonic acid groups has comparable catalytic activity to that of commercial ion-exchange resin Amberlite-120 and the former also showed higher selectivity toward the desired $p, p^{\prime}$ isomer. MCM-48 silica-anchored samples are equally effective for selective synthesis of Bisphenol-A.
\end{abstract}

(c) 2004 Elsevier Inc. All rights reserved.

Keywords: Bisphenol-A synthesis; Heterogeneous catalysis; Immobilization; Mesoporous materials; Sulfonic acid; X-ray absorption spectroscopy

\section{Introduction}

Bisphenol-A is a very important raw material for the production of epoxy resins and other polymers. Customarily it is manufactured by the acid-catalyzed condensation reaction between acetone and phenol (Scheme 1). In industry, ionexchange resins are used worldwide to produce Bisphenol-A in bulk quantities. Use of mineral acid catalysts is avoided for their corrosive nature. However, due to commercial reasons, most of the details about the nature of the catalyst are found only in the patent literature. It has been reported that modified ion-exchange resins, like Amberlyst, show very good activity for Bisphenol-A synthesis with around $90 \%$ selectivity to the desired $p, p^{\prime}$ isomer [1]. However, thermal stability of resin catalysts is very limited and they cannot be used at higher reaction temperatures. Also fouling of the resin catalysts in the reaction medium is a problem. Attempts

\footnotetext{
* Corresponding author.

E-mail address: chem1031@ntu.edu.tw (S. Cheng).

1 Present address: Chemistry Department, Universidad Politecnica de Valencia, Camino de Vera s/n, 46022 Valencia, Spain.
}

have been, therefore, made to develop solid and thermally stable catalyst for Bisphenol-A synthesis.

Solid catalysts like heteropolyacid $\left(\mathrm{H}_{3} \mathrm{PW}_{12} \mathrm{O}_{40}\right)$ supported on K-10 clay [1] and encapsulated in MCM-41 [2] were reported to be active for Bisphenol-A synthesis. Zeolites have been increasingly used in the synthesis of finechemicals due to their high surface area and uniform pore diameter [3-6]. However, pore diameters of zeolites are in the micropore region $(<20 \AA)$ and their suitability for the conversion of larger substrates has been somewhat limited. Singh [7] compared the activities of zeolites like ZSM-5, $\mathrm{H}$-mordenite, and HY with Amberlyst-15 and showed that zeolites with larger pore openings are more selective for the synthesis of Bisphenol-A. However, ion-exchange resins were reported to give higher conversion than zeolites for this process. The discovery of mesoporous molecular sieves of the M41S family, like MCM-41 (hexagonal P6m symmetry), MCM-48 (cubic Ia3d symmetry), and MCM-50 (lamellar), attracted the attention of many research groups as potential catalysts and/or catalyst supports for the conversion of larger substrate molecules [8-10]. They are likely to offer improved reaction selectivity in the conversion of larger substrate molecules in their well-defined channels 


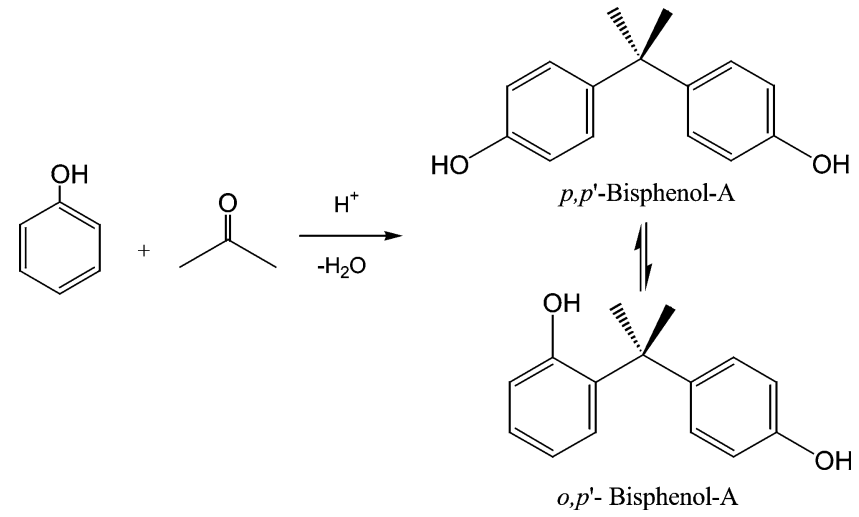

Scheme 1.

with narrow pore-size distribution. However, despite having larger pore dimensions, the acidity of mesoporous materials like Al-MCM-41 is much weaker than that of microporous zeolites [11]. In order to overcome this drawback, the synthesis of hybrid inorganic-organic mesoporous materials with alkylsulfonic acid groups has been reported recently [12-18]. These hybrid materials can be synthesized either by direct one-step synthesis or via secondary silylation with a suitable precursor. Amorphous silica functionalized with sulfonic acid groups was known to behave like a strong acid catalyst [19]. Likewise, $\mathrm{MCM}-\mathrm{SO}_{3} \mathrm{H}$ prepared either by direct synthesis or secondary silylation was reported to be highly efficient in the condensation reactions $[13,14,18]$ and also in the esterification reactions [15-18].

We have shown earlier in a short communication that sulfonic acid-functionalized mesoporous MCM-41 silica catalysts are very effective for Bisphenol-A synthesis at a relatively low reaction temperature [20]. Herein a detailed study of the preparation and catalytic behavior of sulfonic acidfunctionalized MCM-41 and MCM-48 mesoporous silica was presented. Focus was brought on the comparison of their catalytic properties towards Bisphenol-A synthesis. In addition, they were also compared with the catalytic activities of microporous zeolites and commercial sulfonic acid resin Amberlite-120.

\section{Experimental}

\subsection{Catalyst preparation}

Cetyltrimethylammonium bromide (CTABr, 99\%), sodium silicate $\left(\sim 27 \% \quad \mathrm{SiO}_{2}, 14 \% \quad \mathrm{NaOH}\right), \mathrm{H}_{2} \mathrm{SO}_{4}$ $(98+\%)$, and 3-mercaptopropyltrimethoxysilane (3-MPTS, 95\%) were obtained from Aldrich Chemicals. Pure silica MCM-41 was synthesized from a gel with a molar composition of $\mathrm{SiO}_{2}: 0.48 \mathrm{CTA}^{+}: 0.39 \mathrm{Na}_{2} \mathrm{O}: 0.29 \mathrm{H}_{2} \mathrm{SO}_{4}: 110 \mathrm{H}_{2} \mathrm{O}$ according to the method described earlier [21]. Siliceous MCM-48 sample was prepared from a gel with a molar composition of $\mathrm{SiO}_{2}: 0.65 \mathrm{CTA}^{+}: 0.25 \mathrm{Na}_{2} \mathrm{O}: 62 \mathrm{H}_{2} \mathrm{O}$. Ionexchange resin with sulfonic acid functionality Amberlite-
120 (IR-120H) was obtained from Aldrich. Zeolites ZSM-5, beta, and Y were obtained from PQ Corporation, USA.

\subsection{Anchoring of mercapto groups to silica surface}

For functionalization of the silica surface with a sulfonic acid group, mesoporous silica material was reacted with a solution of 3-MPTS according to the method described in the literature $[13,14,18]$. Freshly calcined $\left(525^{\circ} \mathrm{C}, 6 \mathrm{~h}\right)$ MCM-41 or -48 sample $(3.0 \mathrm{~g})$ was evacuated at $150^{\circ} \mathrm{C}$ for $4 \mathrm{~h}$ and then cooled down to room temperature under vacuum and varying amounts of 3-MPTS in dry toluene were introduced. The mixture was refluxed for $4-6 \mathrm{~h}$ and finally the solid was filtered off and washed thoroughly with toluene and dried at $70^{\circ} \mathrm{C}$ in air. Different loadings of the precursor group were achieved by controlling the concentration of the treating solution (3.0-15.0 g of 3-MPTS in $20 \mathrm{ml}$ dry toluene). Some samples were also prepared with uncalcined mesoporous silica materials as the support using the same procedure using ethanol solvent instead of toluene.

\subsection{Oxidation of mercapto groups to thiol groups}

Oxidation of the $-\mathrm{SH}$ groups to the $-\mathrm{SO}_{3} \mathrm{H}$ groups was performed by mild oxidation with $\mathrm{H}_{2} \mathrm{O}_{2}[13,14]$. The solid was stirred for $24 \mathrm{~h}$ at $60^{\circ} \mathrm{C}$ with $35 \% \mathrm{H}_{2} \mathrm{O}_{2}$ solution (an excess of oxidant was used for complete oxidation). The solid was then filtered, washed with water and ethanol, followed by acidification with $0.1 \mathrm{M} \mathrm{H}_{2} \mathrm{SO}_{4}$, and finally washed thoroughly with water to remove all traces of liquid acid. The solid material was finally dried at $60^{\circ} \mathrm{C}$ overnight. The solid was found to be highly hydrophobic due to anchoring of the organic moiety to the surface. The sulfonic acidfunctionalized mesoporous silica samples are designated as MPS- $x$, where $x$ indicates the batch number.

\subsection{Characterization of the catalysts}

Powder X-ray diffraction patterns of the solid catalysts were recorded by a Scintag X1 diffractometer using $\mathrm{Cu}-\mathrm{K}_{\alpha}$ radiation $(\lambda=1.54 \AA)$. Specific surface areas and pore-size distributions of the catalysts were measured by recording the nitrogen adsorption/desorption isotherms at liquid $\mathrm{N}_{2}$ temperature using a Micromeritics ASAP 2100 system. Before measurements, the samples were outgassed at $150^{\circ} \mathrm{C}$ under vacuum $\left(10^{-3}\right.$ Torr $)$ for $8 \mathrm{~h}$ to remove any adsorbed species. Pore-size distribution was determined from the desorption branch of the isotherm by the BJH (Barrett-JoynerHalenda) method using the Halsey equation. Sulfur analysis of the mercaptopropyl-functionalized MCM-41 samples was performed in a Heraus Vario EL analyzer. Thermogravimetric analysis of the thiol-anchored MCM-41 samples was performed in air in a Du Pont 950 instrument with a heating rate of $10^{\circ} \mathrm{C} / \mathrm{min}$. FT infrared spectra were recorded in a Nicolet Magna II 550 spectrophotometer using $\mathrm{KBr}$ pellets. The XANES experiments were performed at beam line 15B at 
the National Synchrotron Radiation Research Center facility at Hsinchu, Taiwan. Standard operating conditions were 1.5 GeV and 120-200 mA. Photon energies were calibrated using the $L$ edge of pure Mo foil.

\subsection{Reaction procedure}

Synthesis of Bisphenol-A was carried out in the liquid phase under atmospheric pressure in a $50-\mathrm{mL}$ glass reactor equipped with a condenser and a magnetic stirrer. Normally, the reactor was charged with phenol (4.7 g) and acetone $(0.58 \mathrm{~g})$. Phenol was taken in excess and no solvent was used. The amount of catalyst used was typically $50 \mathrm{mg}$. The reactants were heated to the desired reaction temperatures $\left(70-125^{\circ} \mathrm{C}\right.$ ) and held there for $24 \mathrm{~h}$. Reaction products were analyzed by gas chromatography (Chrompak 9000) and identified by GC-MAS (HP 6890-5973).

\section{Results and discussion}

A series of mercaptopropyl-incorporated samples were prepared by silylating freshly calcined MCM-41 samples with varying amounts of the anchoring agent in dry toluene. An attempt has also been made to introduce higher amounts of thiol groups by treating uncalcined MCM-41 with an ethanolic solution of the anchoring agent. In the latter case, the hydrophobic portion of the micelles helps to obtain an increased local concentration of 3-MPTS molecules and then mercaptopropyl groups attached to the surface silanol groups through a condensation reaction. The remaining template molecules were removed latter by treatment with $0.05 \mathrm{M}$ ethanolic $\mathrm{H}_{2} \mathrm{SO}_{4}$ solution. Table 1 shows the sulfur content of the catalysts as determined by elemental analysis. It can be seen that with calcined MCM-41 and MCM-48 the maximum sulfur loadings obtained were about 3.5 and $4.8 \mathrm{wt} \%$, respectively. Higher amounts of sulfur can be introduced by treating uncalcined mesoporous silicas with an ethanolic solution of the anchoring agent. Thus, using uncalcined MCM-41 and MCM-48 as high as 7.8 and $15.6 \mathrm{wt} \%$ sulfur, respectively, were introduced. The sulfur content of the samples remained almost unchanged after the removal of the organic template by acid treatment and/or the subsequent oxidation of the thiol groups to sulfonic acid groups by dilute $\mathrm{H}_{2} \mathrm{O}_{2}$. It is also notable that with a similar concentration of the precursor, higher amounts of sulfur can be incorporated into the solid structure of MCM-48 than MCM-41, presumably due to better diffusion of the 3-MPTS molecules in the 3D channels of MCM-48 (see Table 1). In addition, most of these values are higher than that reported by Van Rhijn et al. [13] where an excess of 3-MPTS solution was used.

Thiol functionality is incorporated onto the MCM-41 or -48 surfaces by the condensation reaction between alkoxy groups of the precursor and the surface $-\mathrm{OH}$ groups. As some of the free $-\mathrm{OH}$ groups were lost by condensation among themselves during the calcination process, the amount of thiol group attached to the support was much less than that obtained using uncalcined mesoporous silicas. On the other hand, that a higher amount of sulfur was introduced onto MCM-48 than MCM-41 also implies that MCM-48 contains more surface hydroxyl groups than MCM-41. In fact, MCM-48 has almost a 30\% higher surface area than MCM-41 (see Table 2). However, it is important to note here that the higher loadings of sulfur do not necessarily results in a better catalyst, as it is also necessary to effectively oxidize (vide infra) the thiol groups to the catalytically active sulfonic acid groups.

Powder XRD patterns of the modified samples showed that the hexagonal or cubic structure of the MCM-41 or -48 materials was well preserved after introduction of the thiol functionality. Fig. 1 shows the typical XRD patterns of the thiol-incorporated MCM-41 samples. Intensity of the 100 reflection of the calcined MCM-41 support was slightly decreased after introduction of mercaptopropyl groups. As the loading of the mercaptopropyl group was increased the intensities of the XRD peaks were also further reduced. The long-range ordering was more markedly affected as evident from the disappearance of the 220 reflection in the modified samples. On the other hand, the XRD patterns of the thiolmodified samples prepared with uncalcined support were found to have larger $d$ spacings than those prepared with calcined support. Moreover, the ordered porous structure was

Table 1

Pretreatment conditions and sulfur content of the samples

\begin{tabular}{|c|c|c|c|c|c|c|}
\hline \multirow[t]{2}{*}{ Sample } & \multirow{2}{*}{$\begin{array}{l}\text { Amount of MPTS/g } \\
\text { of solid }\end{array}$} & \multirow[t]{2}{*}{ Support } & \multirow{2}{*}{$\begin{array}{c}\text { Pretreatment } \\
\text { conditions }\end{array}$} & \multicolumn{2}{|c|}{ Sulfur loading $(\mathrm{wt} \%)$} & \multirow{2}{*}{$\begin{array}{c}\text { meq of sulfur } \\
\text { (per g of final solid) }\end{array}$} \\
\hline & & & & Before oxidation & $\overline{\text { After oxidation }}$ & \\
\hline MPS-2 & 1.0 & MCM-41 & Calcined & 2.4 & 2.1 & 0.66 \\
\hline MPS-6 & 3.0 & MCM-41 & Calcined & 2.8 & 2.6 & 0.81 \\
\hline MPS-8 & 5.0 & MCM-41 & Calcined & 3.5 & 3.4 & 1.06 \\
\hline MPS-11 & 2.0 & MCM-41 & Uncalcined & 5.2 & 5.1 & 1.60 \\
\hline MPS-12 & 3.0 & MCM-41 & Uncalcined & 5.5 & 5.2 & 1.63 \\
\hline MPS-9 & 4.0 & MCM-41 & Uncalcined & 6.1 & 5.7 & 1.78 \\
\hline MPS-17 & 5.0 & MCM-41 & Uncalcined & 7.8 & 7.2 & 2.25 \\
\hline MPS-16 & 3.0 & MCM-48 & Calcined & 4.8 & 4.6 & 1.44 \\
\hline MPS-14 & 3.0 & MCM-48 & Uncalcined & 10.2 & 9.7 & 3.03 \\
\hline MPS-13 & 5.0 & MCM-48 & Uncalcined & 15.6 & 14.8 & 4.63 \\
\hline
\end{tabular}


Table 2

Specific surface area, pore volume, and pore diameter of the catalysts

\begin{tabular}{lccc}
\hline Sample & $\begin{array}{c}S_{\mathrm{BET}} \\
\left(\mathrm{m}^{2} / \mathrm{g}\right)\end{array}$ & $\begin{array}{c}V_{\mathrm{p}} \\
\left(\mathrm{cm}^{3} / \mathrm{g}\right)\end{array}$ & $\begin{array}{c}r_{\mathrm{av}} \\
(\AA)\end{array}$ \\
\hline MCM-41 & 1060 & 0.83 & 27 \\
MPS-2 & 1030 & 0.48 & 21 \\
MPS-6 & 750 & 0.45 & 21 \\
MPS-8 & 850 & 0.50 & 22 \\
MPS-11 & 735 & 0.40 & 19 \\
MPS-12 & 690 & 0.39 & 20 \\
MPS-9 & 700 & 0.42 & 19 \\
MPS-17 & 685 & 0.40 & 19 \\
MCM-48 & 1300 & 0.82 & 22 \\
MPS-16 & 1050 & 0.35 & 16 \\
MPS-13 & 740 & 0.40 & 16 \\
MPS-14 & 750 & 0.35 & 16 \\
\hline
\end{tabular}

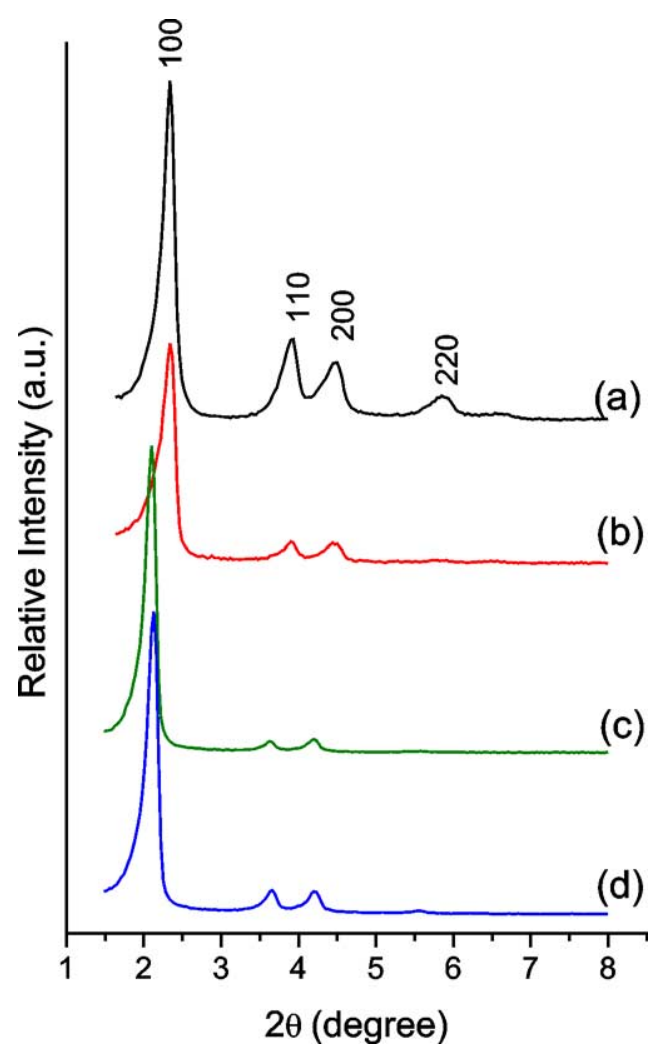

Fig. 1. XRD patterns of different MCM-41 samples: (a) calcined MCM-41, (b) mercaptopropyl anchored on calcined MCM-41 support, (c and d) mercaptopropyl anchored on uncalcined MCM-41 support before and after template extraction.

unaffected by the template-removal process (Fig. 1, c and d). This indicated that the mild acid-extraction process used did not have any adverse effect on the meso-structure of the samples.

Fig. 2 shows the XRD patterns of samples prepared with uncalcined MCM-48 support. The pattern for original MCM-48 was also included for comparison. The slight shift of the 211 reflection in mercaptopropyl-anchored samples was related to the removal of the remaining template mole-

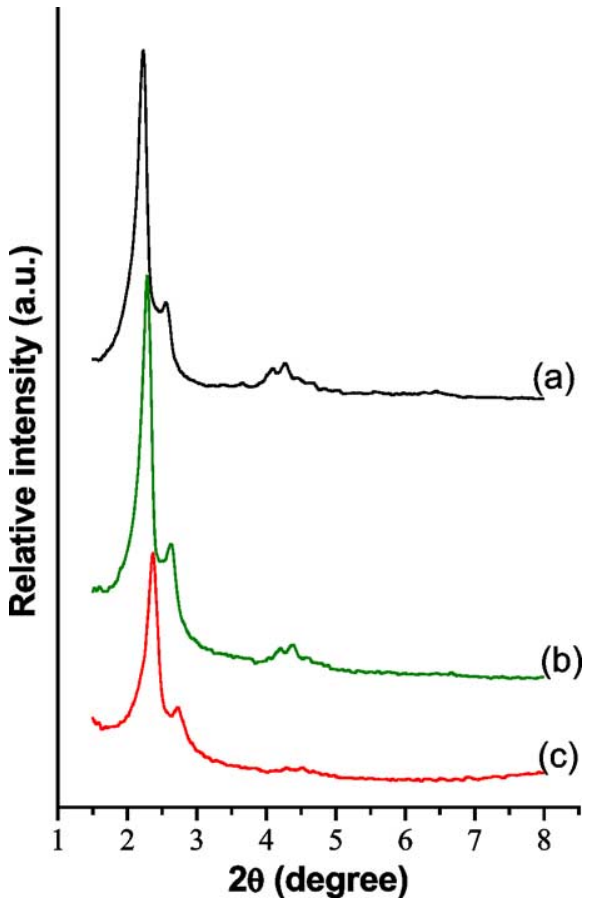

Fig. 2. XRD patterns of (a) uncalcined MCM-48, (b and c) mercaptopropyl-anchored MCM-48 samples MPS-13 and MPS-14, respectively.

cules by acid extraction and subsequent contraction of the unit cells.

A moderate decrease in the specific surface area and pore volume of the samples was observed after the introduction of the thiol groups (Table 2). In comparison to the calcined MCM samples, specific surface area was reduced about $30-40 \%$, while the decrease in pore volume was almost $40-50 \%$. Furthermore, the average pore diameter of the samples shrank by about $6-8 \AA$. Fig. 3 shows the typical $\mathrm{N}_{2}$ adsorption-desorption isotherms and pore-size distributions of a MCM-41 sample and its thiol-modified derivative. It can be seen that the pore-size distribution curve becomes somewhat broader; however, pore-size distribution still remains very narrow with major fractions of pores lying within the $7 \AA$ A region.

Fig. 4 shows a typical thermogram for a thiol-modified sample prepared with uncalcined MCM-41 support. Thermogram for MCM-41 was also included for comparison. In MCM-41, the template starts burning at about $170^{\circ} \mathrm{C}$ and that completes by $300^{\circ} \mathrm{C}$, while for thiol-modified samples the organic groups starts burning at about $300^{\circ} \mathrm{C}$ and that completes by $350^{\circ} \mathrm{C}$. It can also be seen that most of the templates present in the uncalcined MCM-41 support was removed during the anchoring of the thiol groups. The remaining small portion was removed by an acid-extraction process. Also the materials were found to be highly hydrophobic after anchoring of the thiol groups. It was noted that as-synthesized silica MCM-41 showed about 5\% weight loss in $30-150^{\circ} \mathrm{C}$ regions due to water removal. On the other hand thiol-modified MCM-41 showed less than $1 \%$ weight loss before acid extraction in that region. After removal of 


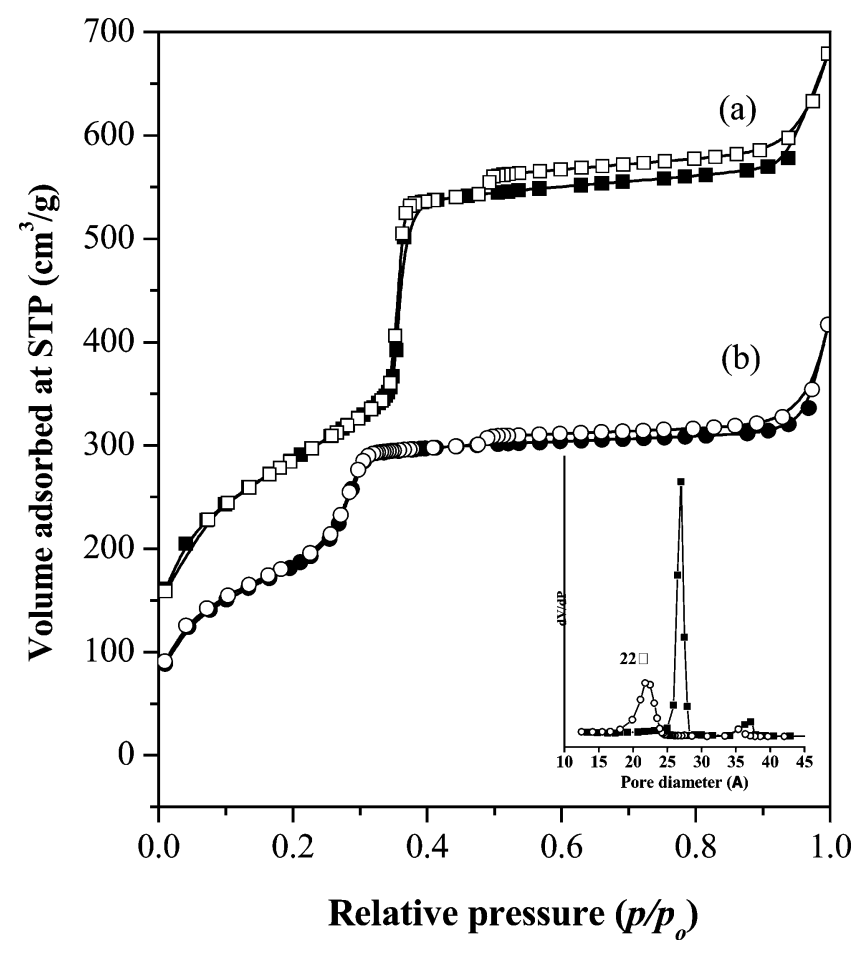

Fig. 3. Nitrogen adsorption-desorption isotherms of (a) calcined MCM-41 and (b) MPS-8 samples; (inset) pore-size distribution patterns of the samples.

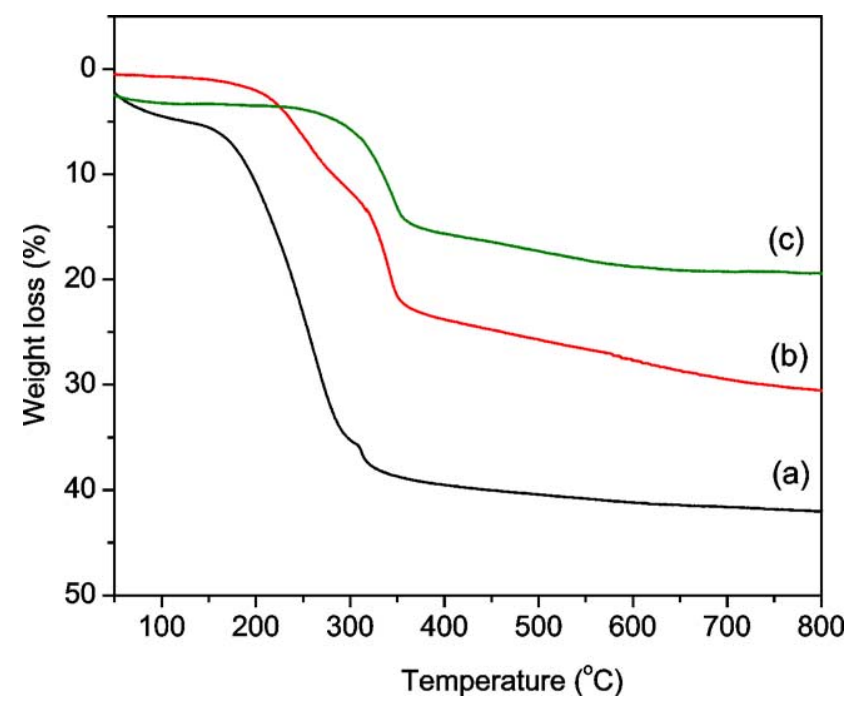

Fig. 4. TGA patterns of (a) as-synthesized MCM-41 and MPS-9 samples (b) before and (c) after acid extraction.

the remaining template molecules by acid extraction the sample showed about 3\% weight loss (Fig. 4, curve c). Samples prepared with MCM-48 silicas also showed a similar behavior.

Infrared spectra of the thiol-anchored MCM-41 samples (Fig. 5) showed two weak bands at about 2930 and $2858 \mathrm{~cm}^{-1}$ characteristic of asymmetric and symmetric stretching vibrations of $-\mathrm{CH}_{2}-$ groups. These bands are absent in calcined MCM-41 and indicate the presence of propyl

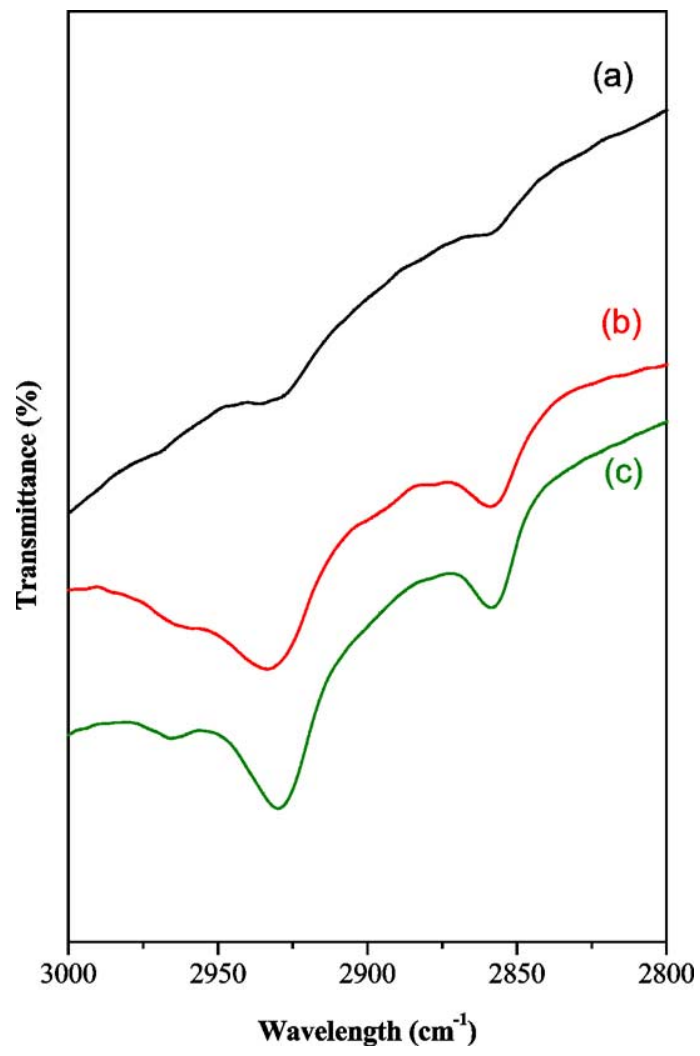

Fig. 5. FT-IR spectra of (a) calcined MCM-41, and MPS-2 (b) before and (c) after oxidation.

tether in the thiol-modified samples. However, no clear information about the presence of sulfur species was obtained from IR studies. Rhijn et al. [13] observed a S-H vibration at about $2575 \mathrm{~cm}^{-1}$. However, their samples were prepared by a different technique. Nevertheless, sulfur $K$-edge XANES studies (vide infra) provided more information about the $\mathrm{S}$ species present in the samples before and after oxidation.

The catalytic functionality of these materials is due to the presence of sulfonic acid groups, which are generated by oxidizing the anchored thiol groups. Thus, in order to obtain good catalytic activity it is essential that the thiol groups are successfully oxidized to the active sulfonic acid groups. To monitor this process, the oxidation states of sulfur were examined by studying the $\mathrm{S} K$-edge XANES spectra of the sample before and after oxidation process. Fig. 6 shows the typical $\mathrm{S} K$-edge XANES spectra of thiol-functionalized sample before and after $\mathrm{H}_{2} \mathrm{O}_{2}$ oxidation. A spectrum for $\mathrm{MgSO}_{4}$ standard was also included for comparison. The energy position $\left(E_{k}\right)$ and intensity of the $S s \rightarrow p$ transition peak (white-line) relate sensitively to the oxidation state of the X-ray-absorbing S atoms [22-25]. Studies with model compounds showed that the $E_{k}$ is related to the formal oxidation states of $\mathrm{S}$ atoms. Thus, for an unoxidized sample containing thiol groups the white-line appeared at $2472 \mathrm{eV}$, corresponding to the sulfur atoms in the reduced state. After oxidation, the white-line shifted to a higher energy side to $2480 \mathrm{eV}$, which corresponds to sulfonic acid groups with 


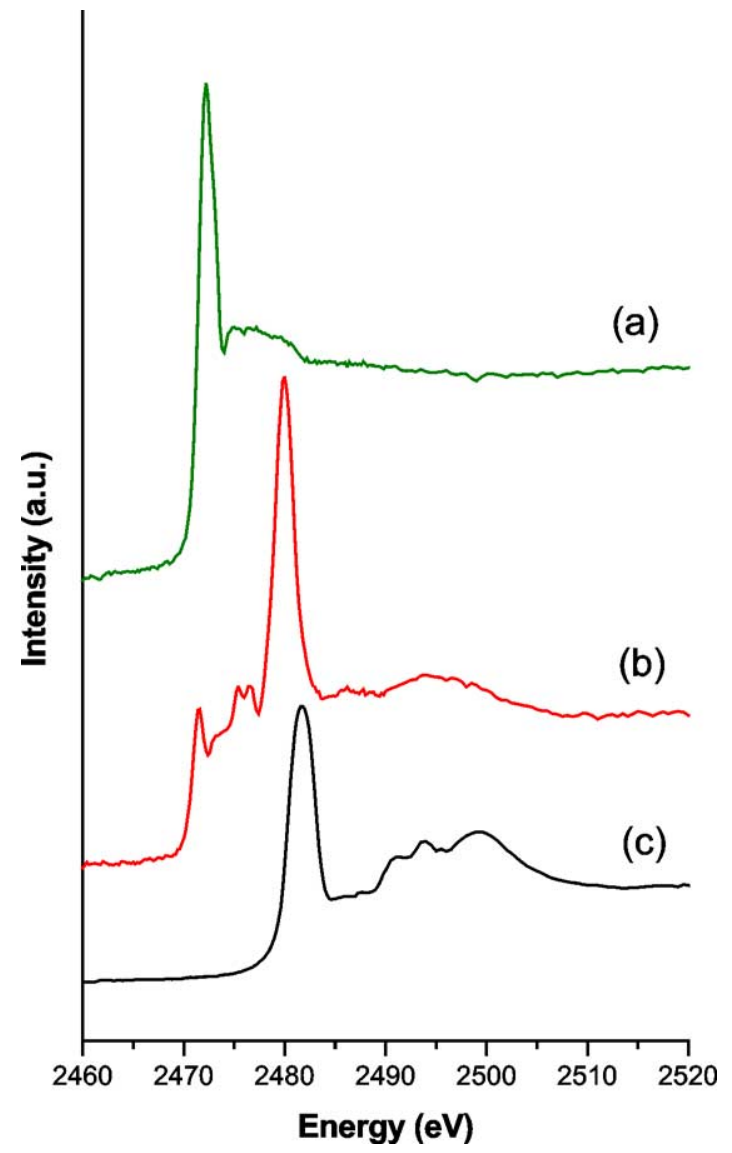

Fig. 6. XANES spectra of mercaptopropyl-anchored MCM-41 silica samples (a) before and (b) after oxidation, and that of (c) $\mathrm{MgSO}_{4}$.

$\mathrm{S}$ in the +5 state $[25,26]$. For $\mathrm{MgSO}_{4}$ with $\mathrm{S}$ in the +6 state, the $E_{k}$ appears at an even higher energy position, $2482 \mathrm{eV}$. It can be seen from Fig. 6 that most of the anchored thiol groups were oxidized to the sulfonic acid groups under the oxidation conditions noted earlier. However, it was noted that for samples having higher sulfur loadings, the oxidation of thiol functionality to sulfonic acid groups was incomplete even after an extended reaction time and other lower valent sulfur species were present in the oxidized samples. Fig. 7 shows the XANES spectra of three samples where sulfur species were oxidized to different degrees. For MPS- 8 with $3.4 \%$ sulfur loadings most of the sulfur species present after oxidation are in the +5 state, whereas for the other two samples with higher sulfur loadings (5.2 and 7.2\%) a part of the sulfur species remained incompletely oxidized. This can be explained that at higher sulfur loadings, there are larger amounts of thiol groups on the surface and the oxidation reaction between two adjacent thiol groups may result in the formation of disulfides $\left(\mathrm{C}_{3}-\mathrm{S}-\mathrm{S}-\mathrm{C}_{3}\right)$, which is relatively inert toward further oxidation. The presence of such disulfide species in the incompletely oxidized samples was also observed by Rhijn et al. [13].

It has been reported that in the $\mathrm{S} K$-edge XANES spectrum the lower valence organic sulfur species (e.g., thiols, sulfides, disulfides) normally appear together as a broad (a)
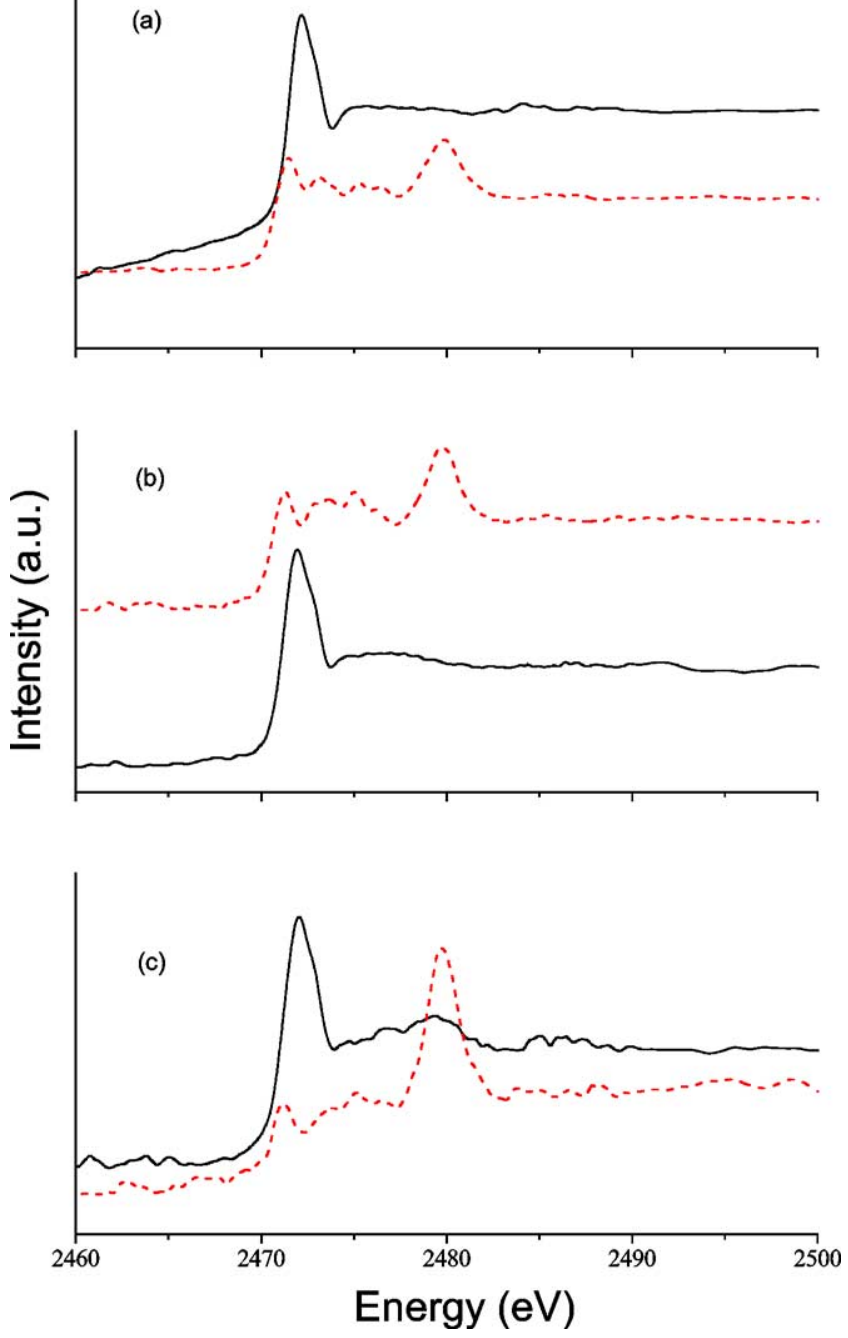

Fig. 7. XANES spectra of (a) MPS-17, (b) MPS-12, and (c) MPS-8 samples before oxidation (solid lines), and after oxidation (dash lines).

band [22]. Comparison between the spectra of unoxidized and the corresponding oxidized samples in Fig. 7 indicates that the extreme left-hand peak appearing at $2471 \mathrm{eV}$ was probably due to the disulfide species. However, in the unoxidized samples this peak was completely masked by the strong peak of the thiol groups appearing at $2472 \mathrm{eV}$. In the spectra of the oxidized samples, measuring the area ratios of the peaks at 2471 (disulfide) and $2480 \mathrm{eV}$ (sulfonic acid) it was observed that the disulfide to sulfonic acid ratio was increased continuously ( 0.13 for MPS- $8,0.40$ for MPS- 12 , and 0.46 for MPS-17). This is quite anticipated as at higher sulfur loadings formation of disulfide species is more likely in the oxidized samples. At higher surface coverage interaction between the neighboring thiol groups is most likely to occur.

\subsection{Catalytic results}

Comparisons of the results of phenol and acetone condensation to Bisphenol-A at $70^{\circ} \mathrm{C}$ on sulfonic acid-function- 
Table 3

Synthesis of Bisphenol-A with different catalysts at $70^{\circ} \mathrm{C}$

\begin{tabular}{lcc}
\hline Catalyst & $\begin{array}{c}\text { Phenol conversion } \\
(\%)\end{array}$ & $\begin{array}{c}\text { Selectivity to } \\
p, p^{\prime} \text {-Bisphenol-A (\%) }\end{array}$ \\
\hline- & 0 & - \\
H-beta $(\mathrm{Si} / \mathrm{Al}=50)$ & 5.2 & 52.8 \\
HY $(\mathrm{Si} / \mathrm{Al}=11)$ & 6.8 & - \\
HZSM-5 $(\mathrm{Si} / \mathrm{Al}=80)$ & 2.7 & 9.9 \\
MPS-8 & 29.6 & 91.7 \\
Amberlite-120 & 35.3 & 89.2 \\
\hline
\end{tabular}

Reaction time $24 \mathrm{~h}$, phenol:acetone molar ratio 5:1.

alized MCM-41 silica and other acidic zeolites are given in Table 3. No conversion of phenol was noted in the absence of the catalyst. Acidic zeolites like H-ZSM-5, H-Y, and H-beta showed negligible activity due to pore-size constraints and also mostly forms undesirable oligomeric products. The sulfonic acid-functionalized MCM-41 silica, MPS-8, showed about $29 \%$ phenol conversion with more than $92 \%$ selectivity toward desired $p, p^{\prime}$-Bisphenol-A. On the other hand, sulfonic acid resin Amberlite-120 showed slightly higher phenol conversion $(35.3 \%$ ) with about $89 \%$ selectivity to the $p, p^{\prime}$ product. Apparently it appears that Amberlite- 120 has a better conversion than MPS-8. However, if the acid sites in the two catalysts were compared we can see that MPS-8 with sulfur loadings of $1.06 \mathrm{meq} / \mathrm{g}$ has a comparable activity as Amberlite-120, which has 4 times (4.4 meq/g) more sulfur loadings. It is noted here that the maximum achievable phenol conversion at a phenol/acetone molar ratio of 5 is $40 \%$.

The other isomer of condensation, $o, p^{\prime}$-Bisphenol-A, was also detected; however, by-products like chroman and trisphenols were not detected at this reaction temperature. Recently, the synthesis of Bisphenol-A over heteropolyacidencapsulated MCM-41 has been reported [2]. 12-Tungstophosphoric acid-encapsulated MCM-41 was found to be catalytic active only at temperatures $120^{\circ} \mathrm{C}$ or above. However, phenol conversion was reported to be much lower than that obtained with MPS samples. In addition, due to the higher reaction temperatures, several by-products like alkylated phenols and chroman derivatives were formed, and the selectivity to $p, p^{\prime}$-Bisphenol-A was less than $70 \%$. Yadav and Kirthivasan [1] compared the activity of different ionexchange resins like Amberlyst-15, -31, -XE-717p, and 12tungstophosphoric acid supported on K-10 clays. Activity of K-10 clay-supported heteropolyacid was found to be similar to that of Amberlyst-31, but much superior selectivity to Bisphenol-A was obtained with the resin catalysts. However, the thermal stability of the resin catalysts was found to be inferior to that of the clay catalysts, which can be used at temperatures as high as $300^{\circ} \mathrm{C}$.

Table 4 shows the phenol conversion and selectivity to $p, p^{\prime}$-Bisphenol-A at $100^{\circ} \mathrm{C}$ for different MCM silica catalysts. Phenol conversion was increased with the increase in reaction temperature, but the activity of the catalysts was not correlated to their sulfur content. Catalysts with higher sul-
Table 4

Synthesis of Bisphenol-A with different mesoporous silica catalysts at $100{ }^{\circ} \mathrm{C}$

\begin{tabular}{lccc}
\hline Catalyst & $\begin{array}{c}\text { meq of sulfur } \\
\text { (per g of final solid) }\end{array}$ & $\begin{array}{c}\text { Phenol } \\
\text { conversion } \\
(\%)\end{array}$ & $\begin{array}{c}\text { Selectivity to } \\
p, p^{\prime} \text {-Bisphenol-A } \\
(\%)\end{array}$ \\
\hline MCM-41 & 0 & 0 & - \\
MPS-6 & 0.81 & 28.6 & 91.2 \\
MPS-8 & 1.06 & 35.3 & 88.6 \\
MPS-12 & 1.63 & 23.5 & 91.6 \\
MPS-9 & 1.78 & 24.8 & 90.8 \\
MPS-17 & 2.25 & 27.5 & 89.8 \\
MPS-16 & 1.44 & 29.1 & 89.7 \\
MPS-14 & 3.03 & 38.2 & 82.6 \\
MPS-13 & 4.63 & 29.3 & 85.4 \\
Amberlite-120 & 4.4 & 39.1 & 86.9 \\
\hline
\end{tabular}

Reaction time $24 \mathrm{~h}$, phenol:acetone molar ratio 5:1.

fur loadings did not generally produce better catalysts. This can be explained by considering the fact that different sulfur species were present in these catalysts after oxidation with $\mathrm{H}_{2} \mathrm{O}_{2}$. Sulfur $K$-edge XANES studies showed that samples containing higher amounts of sulfur are difficult to oxidize completely and a part of the thiol groups was incompletely oxidized or remained at lower valent states, which are catalytically inactive. For maximum catalytic activity, it is necessary that the sulfur species are in the +5 state as sulfonic acid. However, the appearance of a small peak at around $2471 \mathrm{eV}$ in the XANES spectra indicates the presence of disulfide groups. It was noted that when sulfur loadings exceed $1.5 \mathrm{meq} / \mathrm{g}$ solid a part of the sulfur atoms remains in the reduced form even after prolonged oxidation. It appears that at higher surface coverage nearby thiol groups interact easily to form catalytically inactive disulfide species.

It can also be seen from Table 4 that an MCM-48-based MPS-16 catalyst that contains slightly higher sulfur loadings $(1.44(\mathrm{meq} \mathrm{S}) / \mathrm{g})$ than MCM-41-based MPS-8 sample $(1.06(\mathrm{meq} \mathrm{S}) / \mathrm{g})$ showed slightly lower phenol conversion. This again indicates that in samples with higher sulfur loadings a part of thiol groups are incompletely oxidized. Furthermore, like MCM-41-based samples it appears that at higher sulfur loadings oxidation of the thiol groups was more difficult for MCM-48-based samples also. Consequently sample of highest sulfur loading did not give the highest catalytic activity. For example, MPS-13 containing 4.63 (meq S)/g showed $29.3 \%$ phenol conversion, while MPS-14 containing 3.03 (meq S)/g showed $38.2 \%$ phenol conversion. Furthermore, the selectivity to the desired $p, p^{\prime}$-Bisphenol-A was found to be slightly lower over the MCM-48-based catalysts than that over MCM-41-based catalysts. It is probably attributed to the fact that the pore diameter of mesopores in cubic MCM-48 is not straight and not as narrow-ranged as that of hexagonal MCM-41.

To compare the catalytic behavior of the resin and silicabased catalysts, the activity and selectivity of MPS- 8 and Amberlite- 120 at $100{ }^{\circ} \mathrm{C}$ with time-on-stream are given in 


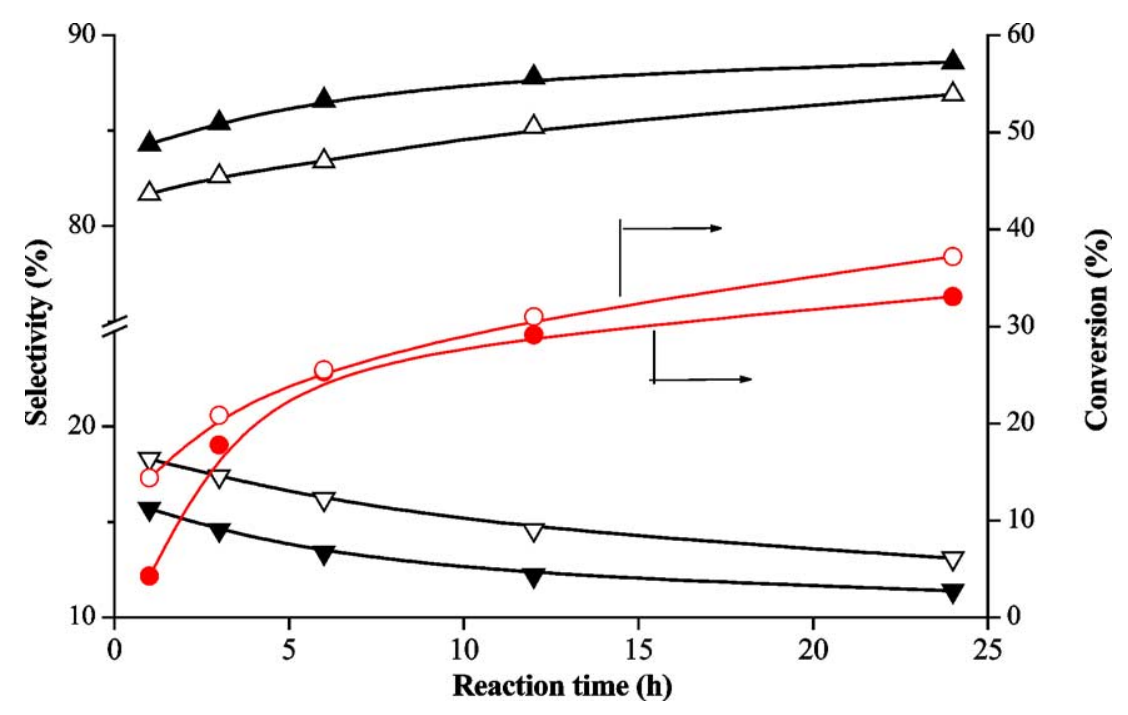

Fig. 8. Selectivity and conversion with time on stream at $100{ }^{\circ} \mathrm{C}\left(\mathbf{\nabla}, \nabla\right.$, selectivity to $o, p^{\prime}$-Bisphenol-A, $\mathbf{\Lambda}, \triangle$, selectivity to $p, p^{\prime}$-Bisphenol-A; and $\bullet, \bigcirc$, conversion; filled symbols for MPS-8, open symbols for Amberlite-120).

Table 5

Synthesis of Bisphenol-A with MPS-8 at different temperatures

\begin{tabular}{cccc}
\hline Temperature & Phenol conversion & \multicolumn{2}{c}{ Selectivity $(\%)$} \\
\cline { 3 - 4 }$\left({ }^{\circ} \mathrm{C}\right)$ & $(\%)$ & $p, p^{\prime}$-Bisphenol-A & $o, p^{\prime}$-Bisphenol-A \\
\hline 70 & 29.6 & 91.7 & 8.3 \\
100 & 35.3 & 88.6 & 11.4 \\
125 & 38.2 & 84.5 & 15.5 \\
150 & 38.6 & 81.4 & 18.6 \\
\hline
\end{tabular}

Reaction time $24 \mathrm{~h}$, phenol:acetone molar ratio 5:1.

Fig. 8. It can be seen that initially silica-based catalysts have a lower conversion than the conventional resin catalysts; however, as the reaction progressed the two catalysts have almost similar activities. Selectivity to the desired $p, p^{\prime}$-Bisphenol-A was found to be always better with thiol-anchored mesoporous MCM-41 catalysts. For both resin and MCM-41-based catalysts, the selectivity to $p, p^{\prime}$ Bisphenol-A decreased slightly as the reaction temperature was raised from 70 to $100^{\circ} \mathrm{C}$, and in the case of resin catalyst small amounts of other by-products like chroman and trisphenols were also detected during the course of the reaction.

Increasing the reaction temperature to $150{ }^{\circ} \mathrm{C}$, phenol conversion achieved with MPS- 8 was $38.6 \%$, which is very close to the maximum activity possible at this phenol/ acetone ratio. However, selectivity to the desired $p, p^{\prime}$ Bisphenol-A was dropped continuously (Table 5) with the increase in reaction temperature. Also small amounts of other by-products like chromans were detected.

Elemental analysis of the used catalyst showed that leaching of sulfur during the catalytic reaction was negligible with MPS samples. For example, the MPS- 8 catalyst contained 3.4 and $3.2 \mathrm{wt} \% \mathrm{~S}$ before and after reaction at $70^{\circ} \mathrm{C}$. However, a slight increase in carbon content indicated the deposition of carbonaceous products during the reaction. The slight decrease in sulfur content observed was mainly due to the increase in weight basis.

In conclusion, sulfonic acid-anchored MCM-41 and MCM-48 have been found to be very effective in the synthesis of $p, p^{\prime}$-Bisphenol-A with very high selectivity at relatively low reaction temperatures. Sulfur $K$-edge XANES studies showed that at low sulfur loadings oxidation of the thiol precursor to the catalytically active sulfonic acid was almost complete. However, at higher sulfur loadings oxidation was incomplete and catalytically inactive lower valent sulfur species like sulfide and disulfides $\mathrm{C}_{3}-\mathrm{S}-\mathrm{S}-\mathrm{C}_{3}$ were present along with sulfonic acid groups.

\section{Acknowledgments}

The authors are grateful to the Chinese Petroleum Corporation, Taiwan, and the Ministry of Education, Taiwan, for providing financial support.

\section{References}

[1] G.D. Yadav, N. Kirthivasan, Appl. Catal. 154 (1997) 29.

[2] K. Nowinska, W. Kaleta, Appl. Catal. A 203 (2001) 91.

[3] M.E. Davis, Micropor. Mesopor. Mater. 21 (1998) 173.

[4] R.A. Sheldon, J.A. Elings, S.K. Lee, H.E.B. Lempers, R.S. Downing, J. Mol. Catal. A 134 (1998) 129.

[5] S. Feast, J.A. Lercher, Stud. Surf. Sci. Catal. 102 (1996) 363.

[6] M.E. Davis, Acc. Chem. Res. 26 (1993) 111.

[7] A.P. Singh, Catal. Lett. 27 (1992) 431.

[8] J.S. Beck, J.C. Vartuli, W.J. Roth, M.E. Leonowicz, C.T. Kresge, K.D. Schmitt, C.T. Chu, D.H. Olson, E.W. Sheppard, S.B. McCullen, J.B. Higgins, J.L. Schlenker, J. Am. Chem. Soc. 114 (1992) 10834.

[9] P.T. Tanev, T.J. Pinnavaia, Science 267 (1995) 865.

[10] X.S. Zhao, G.Q. Lu, G.J. Miller, Ind. Eng. Chem. Res. 35 (1996) 2075.

[11] A. Corma, V. Fornes, M.T. Navarro, J. Perez-Pariente, J. Catal. 148 (1994) 569 
[12] M.H. Lim, C.F. Blanford, A. Stein, Chem. Mater. 10 (1998) 467.

[13] W.M. Van Rhijn, D.E. De Vos, W.D. Bossaert, J. Bullen, B. Wouters, P. Grobet, P.A. Jacobs, Stud. Surf. Sci. Catal. 117 (1998) 183.

[14] W.M. Van Rhijn, D.E. De Vos, B.F. Sels, W.D. Bossaert, P.A. Jacobs, Chem. Commun. (1998) 317.

[15] W.D. Bossaert, D.E. De Vos, W.M. Van Rhijn, J. Bullen, P.J. Grobet, P.A. Jacobs, J. Catal. 182 (1999) 156.

[16] I. Diaz, C. Marquez-Alvarez, F. Mohino, J. Perez-Pariente, E. Sastre, J. Catal. 193 (2000) 295.

[17] I. Diaz, F. Mohino, J. Perez-Pariente, E. Sastre, Appl. Catal. A 205 (2001) 19.

[18] K. Wilson, A.F. Lee, D.J. Macquarrie, J.H. Clark, Appl. Catal. A 228 (2002) 127.
[19] R.D. Badley, W.T. Ford, J. Org. Chem. 54 (1989) 5437.

[20] D. Das, J.-F. Lee, S. Cheng, Chem. Commun. (2001) 2178.

[21] D. Das, C.-M. Tsai, S. Cheng, Chem. Commun. (1999) 473.

[22] A. Vairavamurthy, B. Manowitz, G.W. Luther III, Y. Jeon, Geochim. Cosmochim. Acta 57 (1993) 1619.

[23] A. Vairavamurthy, Spectrochim. Acta A 54 (1998) 2009.

[24] G.P. Huffman, N. Shah, F.E. Huggings, L.M. Stock, K. Chatterjee, J.J. Kilbane II, M.M. Chou, D.H. Buchanan, Fuel 5 (1995) 549.

[25] K. Xia, F. Weesner, W.F. Bleam, P.R. Bloom, U.L. Skyllberg, P.A. Helmke, J. Am. Soil Sci. Soc. 62 (1998) 1240.

[26] G. Sarret, J. Connan, M. Kasrai, G.M. Bancroft, A. Charrie-Duhaut, S. Lemoine, P. Adam, P. Albrecht, L. Eybert-Berard, Geochim. Cosmochim. Acta 63 (1999) 3767. 